\title{
Coagulopatía asociada con COVID y trombosis: oportunidad para cambiar
}

\section{Coagulopathy associated with COVID and thrombosis: an opportunity to change}

\author{
Abraham Majluf-Cruz* \\ Hospital General Regional 1 "Dr. Carlos MacGregor Sánchez Navarro", Unidad de Investigación Médica en Trombosis, Hemostasia y Aterogénesis, \\ Ciudad de México, México
}

El endotelio y la sangre son los dos únicos órganos diseminados en el cuerpo humano y no debiera sorprendernos que siempre estén uno al lado del otro en un maridaje fisiológico exquisitamente regulado. Entre otros pocos factores críticos, la vida humana depende de que la sangre se mantenga líquida; preservar este estado es resultado del funcionamiento óptimo del sistema de coagulación, contrario a la creencia general de que la función esencial de este es formar coágulos. En efecto, la comunicación entre los millones de células de cada humano, su alimentación y oxigenación, la vigilancia inmunológica y el desecho de sustancias indeseables, entre muchas otras funciones, dependen por completo del estado líquido de la sangre.

Por otra parte, del endotelio sano dependen efectos vitales: vasodilatador, antiinflamatorio, anticoagulante, antiangiogénico, antiproliferativo y la regulación siempre apropiada de la permeabilidad vascular; en los estados de disfunción endotelial predominan los efectos vasoconstrictores, protrombóticos, proinflamatorios, proangiogénicos, proproliferativos y se pierde la permeabilidad adecuada. El endotelio sano regula en gran medida los mecanismos inflamatorios y es, por lo tanto, el punto de encuentro de dos sistemas, los cuales hasta hace muy poco tiempo considerábamos separados: coagulación e inflamación. Hoy sabemos que la activación de uno de estos dos se traduce indefectiblemente en la activación del otro, ya que ambos se requieren para la reparación tisular adecuada después de una lesión. Sin embargo, en condiciones patológicas, la activación excesiva y simultánea de los dos sistemas puede tornarse peligrosa. Para resaltar la importancia de esta activación dual, la cual se reconoce cada vez más en el paciente grave, recientemente se acuñó el término "inmunotrombosis". ${ }^{1,2}$

Siendo el endotelio la base del funcionamiento adecuado del sistema de coagulación, este pierde gran parte de sus mecanismos reguladores si existe disfunción endotelial. Por lo tanto, si para mantener la sangre líquida se requiere la regulación exquisita del sistema de coagulación y esta depende de la función endotelial, todo estado de disfunción del endotelio se asocia con la transformación patológica de la sangre a un estado sólido, fenómeno al cual llamamos trombo; su manifestación clínica, la trombosis, es la primera causa de muerte en el humano. ${ }^{3}$ En efecto, los ancianos y los pacientes con enfermedades crónicas no transmisibles (obesidad, tabaquismo, diabetes mellitus, hipertensión arterial, entre otras), desarrollan disfunción endotelial (aunque los últimos mucho más tempranamente) y tienen mayor probabilidad de morir por trombosis.

La infección por el coronavirus SARS-CoV-2 (causante de COVID-19), identificada en diciembre de 2019 en China, ha aumentado exponencialmente en casi todo el mundo. Su tasa de letalidad es baja y la mayoría de los pacientes infectados son asintomáticos; sin embargo, un porcentaje de ellos sufre un tipo de falla uniorgánica (insuficiencia respiratoria) y algunos progresan a una enfermedad sistémica con falla orgánica múltiple. ${ }^{4}$ En algunos casos, la reacción 
inflamatoria alveolar descontrolada induce activación del sistema de coagulación parecida a la coagulación intravascular diseminada clásica. No conocemos las variables inmunológicas, raciales o personales que predisponen a la evolución más agresiva de la infección ni los detalles íntimos de la coagulopatía asociada con COVID-19, pero sabemos que, como consecuencia de esta última, aparece trombosis microvascular diseminada y, por consiguiente, insuficiencia y daño orgánico múltiples, causas fundamentales de la muerte de estos pacientes. ${ }^{4}$

La presencia de factores de riesgo vascular en el paciente con COVID-19 se asocia con la aparición de manifestaciones clínicas más graves. Esto es muy aparente en México ya que la prevalencia de factores de riesgo vascular es muy alta y, por lo tanto, se observa la aparición de infecciones graves en enfermos más jóvenes en comparación con lo que ocurre en Europa, por ejemplo. ${ }^{5,6}$ Una explicación para este fenómeno es que la disfunción endotelial afecta más tempranamente a la población mexicana. Aunado a esto, sabemos que el inicio y mantenimiento de la coagulopatía asociada con COVID-19 están determinados en gran medida por la entrada del virus al endotelio, fenómeno temprano en la infección que se traduce en mayor disfunción endotelial. Un ejemplo claro del papel fundamental de la coagulopatía asociada con COVID-19 es el aumento del dímero $\mathrm{D}$, un marcador de la generación de fibrina, y su lisis subsecuente por la fibrinolisis; el dímero $D$ elevado asociado con COVID-19 es un marcador negativo de coagulopatía, evolución y mortalidad. ${ }^{7}$

Por todo lo anterior, desde la descripción de la coagulopatía asociada con COVID-19 al inicio de la infección, la terapia anticoagulante se convirtió en fundamento del tratamiento. Con diversas terapias se ha intentado contener a COVID-19, pero la mayoría se ha descartado con la publicación de estudios clínicos controlados; el único tratamiento que permanece sin discusión es el anticoagulante.

Desde hace algunos años sabemos que la trombosis es la causa líder tanto de muerte como de discapacidad en el ser humano. ${ }^{8}$ Efectivamente, si sumamos los casos de infarto agudo de miocardio, enfermedad vascular cerebral isquémica, coagulopatía por consumo o tromboembolia pulmonar, entre otras, queda claro este hecho que inicialmente parece dramático en exceso. COVID-19 ha permitido algo que se creía remoto hace tan solo algunos meses: llamar la atención del médico y la sociedad hacia la trombosis.

¿Por qué la trombosis no aparecía hasta ahora en el colectivo mental? Las causas son múltiples. El sistema de coagulación no se enseña en la carrera de medicina a pesar del peso que tendrá sobre la actividad profesional del médico en ciernes. La trombosis no se considera de una trascendencia específica suficiente no solo en el pregrado, sino incluso en el posgrado de las especialidades médicas y, más aún, desgraciadamente, en la formación de hematólogos, quienes deberían ser los expertos en todo lo que atañe a la sangre.

Disponemos de programas sociales, educativos y formativos para que el público desarrolle conciencia acerca de la diabetes mellitus, la obesidad o la hipertensión arterial, pero no existen mecanismos que permitan difundir información en torno a la trombosis en esos niveles. No existen programas institucionales en el sector salud para atacar de frente el problema trombótico, diseñados con la intención de elevar el nivel de conciencia y los conocimientos básicos al respecto, para, consecuentemente, aplicar la tromboprofilaxis o el mejor tratamiento antitrombótico posibles. Por ejemplo, nos preocupa el cáncer, pero obviamos el hecho de que más de $40 \%$ de los casos se complicará o morirá por trombosis. El problema es toral, pero está desatendido. Hoy hablamos cotidianamente de dímero $D$ y heparinas de bajo peso molecular, sin embargo, ¿sabemos qué es realmente el dímero D?, ¿sabemos cómo actúan los antitrombóticos, incluyendo las heparinas? o ¿solo repetimos conceptos asimilados, pero no comprendidos?

Es innegable el impacto de la trombosis sobre la práctica médica actual en casi todas las áreas médicas, incluyendo algunas imposibles de imaginar hace algunos años como la pediatría o la ginecoobstetricia. Aun cuando tenemos a la mano la frialdad y objetividad de los datos epidemiológicos internacionales relativos al impacto de la trombosis, en México existe un divorcio entre la evidencia publicada y la respuesta al problema en todos los niveles médicos y sociales. Estamos llenos de mitos acerca del tratamiento antitrombótico, entre los cuales la hemorragia secundaria a su uso es el más grave.

¿Por qué la resistencia a cambiar el pensamiento y la actitud hacia la trombosis a pesar de la información con la que contamos? Quizá la respuesta sea simple: la hemorragia la vemos, nos aterra, está grabada en nuestra memoria evolutiva como parte de nuestros mecanismos de alerta, matizando aspectos primarios de nuestra vida como, por ejemplo, algunas creencias religiosas. A la trombosis no la vemos; para concebirla se necesita un pensamiento más avanzado, más racional y menos instintivo. Si no lo veo, no lo creo... 
Ciertamente, en el pensamiento médico siempre está presente la hemorragia cuando el asesino está en el lado opuesto de la balanza. COVID-19 nos ha puesto, para bien o para mal, en perspectiva: la hemorragia casi nunca complica esta enfermedad, pero la trombosis mata a la mayoría de los pacientes.

\section{Bibliografía}

1. Vázquez-Garza E, Jerjes-Sánchez C, Joya-Harrison J, Rodríguez D Venous thromboembolism: thrombosis, inflammation, and immunothrombosis for clinicians. J Thromb Thrombolysis. 2017;44:377-385.
2. Gris JC, Pérez-Martin A, Quere I, Sotto A. COVID-19 associated coagulopathy: the crowning glory of thrombo-inflammation concept. Anaesth Crit Care Pain Med. 2020;39:381-382.

3. Kazmi RS, Boyce S, Lwaleed BA. Homeostasis of hemostasis: the role of endothelium. Semin Thromb Hemost. 2015;41:549-555.

4. Wiersinga WJ, Rhodes A, Cheng AC, Peacock SJ, Prescott HC. Pathophysiology, transmission, diagnosis, and treatment of Coronavirus Disease 2019 (COVID-19): a review. JAMA. 2020;324:782-793.

5. Alvarado-Moreno JA, Majluf-Cruz A. COVID-19 and dysfunctional endothelium: the Mexican scenario. Arch Med Res. 2020;51:587-588.

6. Mueller AL, McNamara MS, Sinclair DA. Why does COVID-19 disproportionately affect older people? Aging (Albany NY). 2020;12:9959-9981.

7. Iba T, Levy JH, Connors JM, Warkentin TE, Thachil J, Levi M. The unique characteristics of COVID-19 coagulopathy. Crit Care. 2020;24:360.

8. Raskob GE, Blanco AN, Buller H, Gallus A, Hunt BJ, Hylek EM, et al. Thrombosis: a major contributor to the global disease burden. Arterioscler Thromb Vas Biol. 2014;12:1580-1590. 